\title{
VOLUME MINIMIZING SUBMANIFOLDS IN COMPACT SYMMETRIC SPACES
}

\author{
BY HIROYUKI TASAKI
}

In this note we consider two methods in order to investigate volume minimizing submanifolds in compact symmetric spaces. The first is calibration ([4]) and the second is integral geometry. We can show that certain submanifolds are volume minimizing in their real homology classes using calibrations. A calibration is a closed differential form on a Riemannian manifold which satisfies a certain inequality. A definition of calibrations will be given in Section 1. On the othor hand we can prove that certain submanifolds are volume minimizing in its homotopy classes using integral geometry. We shall use a generalized Poincaré's formula in Riemannian homogeneous spaces given by Howard [7].

\section{Calibrations}

Let $M$ be a Riemannian manifold with a closed $p$-form $\phi$ on $M$ which satisfies the following inequality:

$$
\left.\phi\right|_{\xi} \leq \operatorname{vol}_{\xi}
$$

for any oriented tangent $p$-plane $\xi$ on $M$. Such a form $\phi$ is called a calibration. Then any compact oriented $p$-dimensional submanifold $N$ in $M$ with the property:

$$
\left.\phi\right|_{N}=\operatorname{vol}_{N}
$$

is volume minimizing in $M$, that is,

$$
\operatorname{vol}(N) \leq \operatorname{vol}\left(N^{\prime}\right)
$$

for any compact oriented $p$-dimensional submanifold $N^{\prime}$ such that $[N]=\left[N^{\prime}\right]$ in the homology group $H_{p}(M ; \mathbf{R})$. We say that $\phi$ calibrates $N$. Using Stokes' theorem we get

$$
\operatorname{vol}(N)=\int_{N} \operatorname{vol}_{N}=\int_{N} \phi=\int_{N^{\prime}} \phi \leq \int_{N^{\prime}} \operatorname{vol}_{N^{\prime}}=\operatorname{vol}\left(N^{\prime}\right) .
$$

The equality holds if and only if $\left.\phi\right|_{N^{\prime}}=\operatorname{vol}_{N^{\prime}}$.

The fundamental 2-form of a Kählar manifold is one of important examples of clibrations. It satisfies Wirtnger's inequality, which is stated as follows. Let $M$ be a Kähler manifold with fundamental 2-form $\omega$. Then

$$
\left.\frac{1}{k !} \omega^{k}\right|_{\xi} \leq \operatorname{vol}_{\xi}
$$

for $1 \leq k \leq \operatorname{dim}_{C} M$ and any oriented tangent $2 k$-plane $\xi$ on $M$. Therefore $\frac{1}{k !} \omega^{k}$ is a calibration on $M$. The equality holds if any only if $\xi$ is a complex plane with a canonical

Received May 31, 1993. 
orientation. So it follows that a compact Kähler submanifold of a Kähler manifold is volume minimizing in its real homology class.

A similar inequality on a quaternionic Kähler manifold is given by Berger [1]. As a conclusion a compact quaternionic submanifold in it is volume minimizing in its real homology class. Quaternionic symmetric spaces are classified by Wolf [16] and quaternionic submanifolds in them are investigated in [14].

We assume that $M$ is a compact symmetric space. We denote by $G$ the identity component of the group of all isometries of $M$. We take and fix a point $x$ in $M$ and denote by $K$ the isotropy subgroup of $G$ at $x$, that is,

$$
K=\{g \in G \mid g(x)=x\} .
$$

Then $K$ acts linearly on the tangent space $T_{x}(M)$ to $M$ at $x$ in the following way.

$$
k \cdot v=(d k)_{x}(v) \quad \text { for } v \in T_{x}(M) .
$$

The following isomorphisms are important for constructing calibrations on compact symmetric spaces.

$$
\begin{aligned}
& H^{p}(M ; \mathbf{R}) \\
\cong & \{\text { harmonic } p \text {-forms on } M\} \\
= & \{\text { parallel } p \text {-forms on } M\} \\
= & \{G \text {-invariant differential } p \text {-forms on } M\} \\
\cong & \left\{K \text {-invariant alternating } p \text {-forms on } T_{x}(M)\right\}
\end{aligned}
$$

We denote by $\mathfrak{g}$ and $\mathfrak{k}$ the Lie algebras of $G$ and $K$ respectively. Let

$$
\mathfrak{g}=\mathfrak{k}+\mathfrak{p}
$$

be a canonical direct sum decomposition of $\mathfrak{g}$, which satisfies

$$
[\mathfrak{k}, \mathfrak{p}] \subset \mathfrak{p}, \quad[\mathfrak{p}, \mathfrak{p}] \subset \mathfrak{k} .
$$

Since the quotient space $G / K$ are diffeomorphic to $M$, we can identify $\mathfrak{p}$ and the tangent space $T_{x}(M)$. The action of $K$ on $T_{x}(M)$ is nothing but the adjoint action of $K$ on $\mathfrak{p}$ through the identification. Hence we get

$$
H^{p}(M ; \mathbf{R}) \cong\left\{\operatorname{Ad}_{G}(K) \text {-invariant alternating } p \text {-forms on } \mathfrak{p}\right\} \text {. }
$$

If $\omega$ is an $\operatorname{Ad}_{G}(K)$-invariant alternating $p$-form on $\mathfrak{p}$, then we can extend $\omega$ to a parallel differential $p$-form $\bar{\omega}$ on $M$. If $\omega$ satisfies an inequality $\left.\omega\right|_{\xi} \leq \operatorname{vol}_{\xi}$ for any oriented $p$-plane $\xi$ in $\mathfrak{p}$, then the same inequality holds at each point in $M$, that is, $\bar{\omega}$ is a calibration on $M$.

Dao Chong Thi [2] constructed a calibration of degree 3 on $S U(n)$ and proved that $S U(2)$ in $S U(n)$ is volume minimizing in its real homology class. Moreover I constructed a calibration $\alpha$ of degree 3 on a compact simple Lie group and proved that a 3 -dimensional compact Lie subgroup $G_{1}$ associated with the highest root is volume minimizing in its real homology class in [13]. Instead of giving an explicit definition of $G_{1}$, we give some examples of $G_{1}$. In the case that $G=S U(n), G_{1}$ is $S U(2)$ naturally embedded in $S U(n)$. In the case that $G=S O(n), G_{1}$ is $S U(2)$ naturally embedded in $S O(4)$. In the case that $G=S p(n), G_{1}$ is $S p(1)$ naturally embedded in $S p(n)$.

We can see that the first conjugate locus $F$ of $G$ determines a closed current of codimension 3 , using the stratified structure of $F$, which is investigated by Sakai [10] 
and Takeuchi [12]. We denote by $*$ the Hodge star operator on $G$. $* \alpha$ is a calibration on $G$ and calibrates the first conjugate locus of $G$. In particular, the current determined by $F$ is mass minimizing in its real homology class. It is a general result that $* \psi$ is a calibration if and only if $\psi$ is a calibration.

This method is useful for some of the other compact symmetric spaces. For example, the following theorems hold.

THEOREM $1.1([3]) . \quad S O(m) / S O(p) \times S O(m-p)$ is volume minımızıng in its real homology class in $S O(n) / S O(p) \times S O(n-p)$, if $n>m$ and if $p$ is an even integer which is greater than or equal to 4.

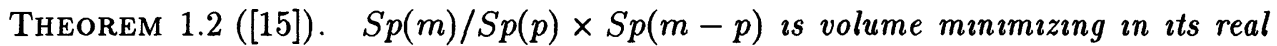
homology class in $S p(n) / S p(p) \times S p(n-p)$, if $n>m$.

\section{Integral geometry}

In this section we shall consider an estimate of the volumes of submanifolds from below in some compact symmetric spaces from a viewpoint of integral geometry under cut loci. As an application of this estimate we can prove that Helgason spheres in certain compact symmetric spaces are volume minimizing in the class of submanifolds whose inclusion maps are not null homotopic.

We review integral geometry, especially Poincaré's formula. We assume that $\sigma, \tau$ are curves with finite lengths in the plane $\mathbf{R}^{2}$ and $M\left(\mathbf{R}^{2}\right)$ is the group of isometries of $\mathbf{R}^{2}$ with Haar measure $\mu_{M\left(\mathbf{R}^{2}\right)}$. Then the following formula holds.

$$
\int_{M\left(\mathbf{R}^{2}\right)} \sharp(g \sigma \cap \tau) d \mu_{M\left(\mathbf{R}^{2}\right)}(g)=4 L(\sigma) L(\tau),
$$

where $L(\sigma)$ is the length of $\sigma$. This is called Poincarés formula. Many mathematicians Santalo, Chern, Gelfand and others have extended and generalized this formula. Among them R. Howard generalized it to the case of Riemannian homogeneous spaces. We take submanifolds $M$ and $N$ in a Riemannian homogeneous space $G / K$ and assume $\operatorname{dim} M+\operatorname{dim} N=\operatorname{dim}(G / K)$. The following formula is a special case of that given by Howard [7].

$$
\int_{G} \sharp(M \cap g N) d \mu_{G}(g)=\int_{M \times N} \sigma_{K}\left(T_{x}^{\perp}(M), T_{y}^{\perp}(N)\right) d \mu_{M \times N}(x, y),
$$

where $\sigma_{K}$ is the average of angles between tangent subspaces under the group action. We shall use this formula later.

Next we give a definition of the cut locus in a compact connected Riemannian manifold. We assume that $M$ is a compact connected Riemannian manifold and denote by $\operatorname{Exp}_{x}$ the exponential map at a point $x$ in $M$. We consider the geodesic $t \mapsto \gamma_{X}(t)=$ $\operatorname{Exp}_{x} t X$ with unit initial direction $X . \gamma_{X}$ is a minimizing geodesic joining $x$ and $\gamma_{X}(t)$ for a small $t$, but there exists the last point $\gamma_{X}\left(t_{0}(X)\right)$ where this minimizing property holds. We call such a point the cut point of $x$ along $\gamma_{X}$. The cut locus $C_{x}(M)$ of $x$ in $M$ is defined by the set of all cut points of $x$.

$$
C_{x}(M)=\left\{\gamma_{X}\left(t_{0}(X)\right)\left|X \in Y_{x}(M),\right| X \mid=1\right\}
$$


Then $B_{x}(M)=M-C_{x}(M)$ is an open ball and $M=B_{x}(M) \cup C_{x}(M)$. By this decomposition we obtain the following lemma.

LEMMA 2.1. Let $N$ be a subset of $M$ whose inclusıon map is not null homotopic. Then $N \cap C_{x}(M)$ is not empty for any $x$ in $M$.

From now on we assume that $M=G / K$ is a compact simply connected irreducible symmetric space. The class of compact symmetric spaces contains all compact simple Lie groups, all kinds of Grassmann manifolds and etc. The cut locus in $M$ is not a submanifold in general but has a nice structure. There is a submanifold $C_{x}^{0}(M)$ which is open and dense in $C_{x}(M)$ and the singular set $C_{x}(M)-C_{x}^{0}(M)$ is stratified by finitely many submanifolds whose dimensions are less than $\operatorname{dim}\left(C_{x}^{0}(M)\right)$. This structure theorem was obtained by Helgason [6], Sakai [10] and Takeuchi [12]. Now we give a definition of Helgason spheres in the symmetric space $M$. A totally geodesic sphere of maximal dimension with maximal sectional curvature in $M$ is called a Helgason sphere. Helgason [5] proved that all of such spheres are conjugate under the action of the identity component of the group of isometries of $M$. So we take and fix one Helgason sphere $S$ containing the origin $o$ in $M$.

The Helgason sphere has a maximal sectional curvature. So you may expect that its volume is minimizing in some sense. Ohnita [9] proved that the Helgason sphere is weakly stable as a minimal submanifold, that is, its second variation of the volume is positive semidefinite. Moreover it is proved that the Helgason spheres are volume minimizing in their real homology classes in some symmetric spaces. For example, $G_{1}$ mentioned in Section 1 in a compact simple Lie group is a Helgason sphere.

A calibration is a representative of the de Rham cohomology. So we cannot change the coefficient of homology and cohomology to another ring when we use calibrations. If the real homology class represented by the Helgason sphere vanishes, then we cannot use calibrations to consider the volume minimizing property of it. In $M=S O(n+k) / S O(n) \times$ $S O(k)(n, k \geq 3)$, the dimension of the Helgason sphere is 2 , but $H_{2}(M ; \mathbf{R})=\{0\}$. In order to consider the volume minimizing property of the Helgason sphere in such a case we use the cut locus.

We take a canonical direct sum decomposition

$$
\mathfrak{g}=\mathfrak{k}+\mathfrak{p}
$$

of the Lie algebra $\mathfrak{g}$ of $G$ and identify $\mathfrak{p}$ with $T_{o}(M)$. We denote by $\mathfrak{s}$ the tnagent space to $S$ at $o$ and by $P_{S}: \mathfrak{p} \rightarrow \mathfrak{s}$ the orthogonal projection from $\mathfrak{p}$ to $\mathfrak{s}$ and $p=\operatorname{dim} S$. In the formula

$$
\int_{G} \sharp\left(N \cap g C_{o}\right) d \mu_{G}(g)=\int_{N \times C_{o}} \sigma_{K}\left(T_{x}^{\perp}(N), T_{y}^{\perp}\left(C_{o}\right)\right) d \mu_{N \times C_{o}(x, y)}
$$

we consider an estimate of $\sigma_{K}$. For a $p$-dimensional subspace $V$ with orthonormal basis $V_{1}, \ldots, V_{p}$ in $\mathfrak{p}$, we obtain

$$
\sigma_{K}\left(V^{\perp}, T_{y}^{\perp}\left(C_{o}\right)\right)=\int_{K}\left|P_{s}\left(\operatorname{Ad}(k) V_{1}\right) \wedge \cdots \wedge P_{S}\left(\operatorname{Ad}(k) V_{p}\right)\right| d \mu_{K}(k) .
$$

In order to estimate the above integral formula we consider the following four conditions concerning the cut locus and the Helgason sphere in $M$. 
1. There exists a positive constant $C$ such that

$$
\int_{K}\left|P_{s}(\operatorname{Ad}(k) v)\right|^{p} d \mu_{K}(k)=C|v|^{p} \quad \text { for all } v \in \mathfrak{p} .
$$

2. For any $k$ in $K$, the map $P_{s}: \operatorname{Ad}(k) \mathfrak{s} \longrightarrow \mathfrak{s}$ is conformal, that is, there is a nonnegative constant $C_{k}$ which satisfies

$$
\left(P_{s}(\operatorname{Ad}(k) u), P_{s}(\operatorname{Ad}(k) v)\right)=C_{k}(u, v) \quad \text { for } u, v \in \mathfrak{s} .
$$

3. $\sharp\left(S \cap g C_{o}(M)\right)=1$ for almost all $g \in G$.

4. The inclusion map of $S$ to $M$ is not null homotopic.

Then we obtain the following theorem.

THEOREM 2.2. Let $N$ be a p-dimensional submanifold of finite volume in $M$. If (1) is satısfied, then we obtain

$$
\int_{G} \sharp\left(N \cap g C_{o}(M)\right) d \mu_{G}(g) \leq C \operatorname{vol}(N) \operatorname{vol}\left(C_{o}(M)\right) .
$$

If (1)-(4) are satısfied, then

$$
\operatorname{vol}(S) \leq \frac{1}{C \operatorname{vol}\left(C_{o}\right)} \int_{G} \sharp\left(N \cap g C_{o}\right) d \mu_{G}(g) \leq \operatorname{vol}(N)
$$

for $N$ whose inclusion map is not null homotopıc. In partıcular, a Helgason sphere is volume minımızing in the class of submanifolds of dimension $p$ whose inclusion maps are not null homotopic.

It is known that a compact symmetric space of rank one satisfies all of (1)-(4). Therefore the conclusions of Theorem 2.2 hold for a compact symmetric space $M$ of rank one.

If $M$ is a compact irreducible Hermitian symmetric space, a Helgason sphere in $M$ is isomorphic to a complex projective line. We can show all of (1)-(4) for $M$, using some results on compact Hermitian symmetric spaces. In this case the conclusions of Theorem 2.2 also hold.

We consider compact quaternionic symmetric spaces. The class of them contains $S O(n+4) / S O(4) \times S O(n), P^{n}(\mathbf{H})=S p(n+1) / S p(1) \times S p(n), S U(n+2) / S(U(2) \times U(n))$,

If $M$ is a compact quaternionic symmetric space except quaternionic projective spaces, a Helgason sphere in $M$ is isometric to a standard 2-sphere and has some nice properties to prove (1)-(4). Although I asserted that it satisfies (1)-(4), I found a gap of my proof of (4) after the workshop and have not yet given a complete proof of it. The reason why we treat the compact quaternionic summetric spaces is that they are simply connected and the second homotopy groups of them are isomorphic to $\mathbf{Z}_{2}$ except for $P^{n}(\mathbf{H})$ and $S U(n+2) / S(U(2) \times U(n))$. So we cannot consider the volume minimizing property of the Helgason sphere in its real homology class.

We give an outline of the proof of Theorem 2.2.

$$
\begin{aligned}
& \sigma_{K}\left(V^{\perp}, T_{y}^{\perp}\left(C_{o}\right)\right) \\
= & \int_{K}\left|P_{s}\left(\operatorname{Ad}(k) v_{1}\right) \wedge \cdots \wedge P_{s}\left(\operatorname{Ad}(k) V_{p}\right)\right| d \mu_{K}(k)
\end{aligned}
$$




$$
\begin{aligned}
& \leq \int_{K} \mid P_{s}\left(\operatorname{Ad}(k) v_{1}|\cdots| P_{s}\left(\operatorname{Ad}(k) v_{p}\right) \mid d \mu_{K}(k)\right. \\
& \leq \prod_{i=1}^{p}\left(\int_{K}\left|P_{s}\left(\operatorname{Ad}(k) v_{i}\right)\right|^{p} d \mu_{K}(k)\right)^{1 / p}
\end{aligned}
$$

by Hölder's inequality. If (1) is satisfied, then we get

$$
\int_{G} \sharp\left(N \cap g C_{o}\right) d \mu_{G}(g) \leq C \operatorname{vol}(N) \operatorname{vol}\left(C_{o}\right) .
$$

Now we assume that (1)-(4) are satisfied. If $N=S$, then the above equalities hold and we obtain

$$
\operatorname{vol}(G)=\int_{G} \sharp\left(S \cap g C_{o}\right) d \mu_{G}(g)=C \operatorname{vol}(S) \operatorname{vol}\left(C_{o}\right) .
$$

Therefore $\operatorname{vol}(S)=\frac{1}{C \operatorname{vol}\left(C_{o}\right)}$. If the inclusion map of $N$ is not null homotopic, then by Lemma 2.1 we get

$$
\operatorname{vol}(G) \leq \int_{G} \sharp\left(N \cap g C_{o}\right) d \mu_{G}(g)
$$

and

$$
\operatorname{vol}(S) \leq \frac{1}{C \operatorname{vol}\left(C_{o}\right)} \int_{G} \sharp\left(N \cap g C_{o}\right) d \mu_{G}(g) \leq \operatorname{vol}(N) .
$$

Recently Le Hong Van [8] showed that a certain sub-Grassmann manifold in a Grassmann manifold is volume minimizing in its integral homology class using the method of integral geometry.

\section{REFERENCE}

[1] M. Berger, Du côté de chez Pu, Ann. scient. Éc. Norm. Sup., 5 (1972), 1-44.

[2] Dao Chong Thi, Minimal real currents on compact Riemannian manifolds, Math. USSR Izv. 11 (1977), 807-820.

[ 3 ] H. Gluck, F. Morgan and W. Ziller, Calibrated geometries in Grassmannian manifolds, Comm. Math. Helv. 64 (1989), 256-268.

[4] R. Harvey and H. B. Lawson, Jr., Calibrated geometries, Acta Math., 148 (1982), 47-157.

[ 5 ] S. Helgason, Totally geodesic spheres in compact symmetric spaces, Math. Ann., 165 (1966), 309-317.

[6] S. Helgason, Differential geometry, Lie groups, and symmetric spaces, Academic Press, New York, 1978.

[ 7 ] R. Howard, Classical integral geometry in Riemannian homogeneous spaces, Contemporary Math., 63 (1987), 179-204.

[ 8 ] Le Hong Van, Application of integral geometry to minimal surfaces, International J. Math., 4 (1993), 89-111.

[9] Y. Ohnita, On stability of minimal submanifolds in compact symmetric spaces, Compositio Math., 64 (1987), 157-189.

[10] T. Sakai, On the structure of cut loci in compact Riemannian symmetric spaces, Math. Ann., 235 (1978), 129-148.

[11] S. Salamon, Quaternionic Kähler manifolds, Invent. Math., 67 (1982), 143-171.

[12] M. Takeuchi, On conjugate loci and cut loci of compact symmetric spaces I, Tsukuba J. Math., 2 (1978), 35-68. 
[13] H. Tasaki, Certain minimal or homologically volume minimizing submanifolds in compact symmetric spaces, Tsukuba J. Math., 9 (1985), 117-131.

[14] H. Tasaki, Quaternionic submanifolds in quaternionic symmetric spaces, Tôhoku Math. J., 38 (1986), 513-538.

[15] H. Tasaki, Calibrated geometries in quaternionic Grassmannians, Osaka J. Math. 25 (1988), 591-598.

[16] J.A. Wolf, Complex homogeneous contact manifolds and quaternionic symmetric spaces, J. Math. Mech., 14 (1965), 1033-1047.

INSTITUTE OF MATHEMATICS

UNIVERSITY OF TSUKUBA

TSUKUBA, IBARAKI, 305, JAPAN

e-mail: tasaki@math.tsukuba.ac.jp 